\title{
Asymptotic Stability of Traveling Waves for Scalar Viscous Conservation Laws with Non-convex Nonlinearity
}

\author{
Akitaka Matsumura ${ }^{1}$, Kenji Nishihara ${ }^{2}$ \\ ${ }^{1}$ Department of Mathematics, Osaka University, Osaka 560, Japan \\ 2 School of Political Science and Economics, Waseda University, Tokyo 169-50, Japan
}

Received: 15 September 1993

\begin{abstract}
The asymptotic stability of traveling wave solutions with shock profile is considered for scalar viscous conservation laws $u_{t}+f(u)_{x}=\mu u_{x x}$ with the initial data $u_{0}$ which tend to the constant states $u_{ \pm}$as $x \rightarrow \pm \infty$. Stability theorems are obtained in the absence of the convexity of $f$ and in the allowance of $s$ (shock speed $)=f^{\prime}\left(u_{ \pm}\right)$. Moreover, the rate of asymptotics in time is investigated. For the case $f^{\prime}\left(u_{+}\right)<s<f^{\prime}\left(u_{-}\right)$, if the integral of the initial disturbance over $(-\infty, x)$ is small and decays at the algebraic rate as $|x| \rightarrow \infty$, then the solution approaches the traveling wave at the corresponding rate as $t \rightarrow \infty$. This rate seems to be almost optimal compared with the rate in the case $f=u^{2} / 2$ for which an explicit form of the solution exists. The rate is also obtained in the case $f^{\prime}\left(u_{ \pm}\right)=s$ under some additional conditions. Proofs are given by applying an elementary weighted energy method to the integrated equation of the original one. The selection of the weight plays a crucial role in those procedures.
\end{abstract}

\section{Introduction}

We consider the Cauchy problem for scalar viscous conservation laws:

$$
\begin{gathered}
u_{t}+f(u)_{x}=\mu u_{x x}, \quad x \in \mathbf{R}, t>0, \\
u(0, x)=u_{0}(x), \quad x \in \mathbf{R},
\end{gathered}
$$

where $f \in C^{2}$ under consideration, $\mu$ is a positive constant and the initial data $u_{0}(x)$ is asymptotically constant as $x \rightarrow \pm \infty$ :

$$
u_{0}(x) \rightarrow u_{ \pm} \quad \text { as } \quad x \rightarrow \pm \infty
$$

Let Eq. (1.1) admit traveling wave solutions with shock profile such that

$$
u=U(x-s t) \equiv U(\xi), \quad U(\xi) \rightarrow u_{ \pm} \quad \text { as } \quad \xi \rightarrow \pm \infty,
$$

where the constants $u_{ \pm}$and $s$ (shock speed) satisfy the Rankine-Hugoniot condition

$$
-s\left(u_{+}-u_{-}\right)+f\left(u_{+}\right)-f\left(u_{-}\right)=0,
$$


and the generalized shock condition

$$
h(u) \equiv-s\left(u-u_{ \pm}\right)+f(u)-f\left(u_{ \pm}\right)\left\{\begin{array}{cll}
<0 & \text { if } & u_{+}<u<u_{-} \\
>0 & \text { if } & u_{-}<u<u_{+}
\end{array} .\right.
$$

It is noted that the condition (1.6) implies

$$
f^{\prime}\left(u_{+}\right) \leqq s \leqq f^{\prime}\left(u_{-}\right)
$$

and that, especially when $f^{\prime \prime}>0$, the condition (1.6) is equivalent to

$$
f^{\prime}\left(u_{+}\right)<s<f^{\prime}\left(u_{-}\right) \text {, }
$$

which is well-known as Lax's shock condition (Lax [5]).

When $f^{\prime \prime}>0$ and so $f^{\prime}\left(u_{+}\right)<s<f^{\prime}\left(u_{-}\right)$, the stability theorems of the traveling wave solutions have been investigated by Il'in and Oleinik [1], Kawashima and Matsumura [3], Nishihara [7] and etc. Recently, in the absence of $f^{\prime \prime}>0$ the stability theorems have been also investigated by Kawashima and Matsumura [4], Jones, Gardner and Kapitula [2], Mei [6]. See also Osher and Ralston [8], Weinberger [9].

Especially, for $f \in C^{2}$ and $f^{\prime}\left(u_{+}\right)<s<f^{\prime}\left(u_{-}\right)$Jones et al. [2] have obtained the stability of traveling wave solutions and the speed of asymptotics, which are based on the spectral analysis. But its rate of asymptotic speed is less sufficient than that of Kawashima and Matsumura [3] for $f^{\prime \prime}>0$. When $f$ has only one inflection point, the stability theorem has been obtained by Kawashima and Matsumura [4] including the system case and the rate of speed by Mei [6], both of which are due to the energy method. Mei [6] also has treated the case of $s=f^{\prime}\left(u_{ \pm}\right)$for the first time.

Our purpose is to show the stability and the rate of asymptotic speed for any $f \in C^{2}$ which satisfy the generalized shock condition (1.6) only. Even when $f^{\prime}\left(u_{+}\right)<$ $s<f^{\prime}\left(u_{-}\right)$, our rate is sharper than that of Kawashima and Matsumura [3], and seems to be almost optimal from the viewpoint of the optimality in Nishihara [7] for $f=u^{2} / 2$. Our results are given by a rather elementary weighted energy method, but the weight function will be suitably taken.

Our plan of this paper is as follows. After stating the notations, the main theorems will be given in Sect. 2. In Sect. 3 we reformulate our problem and state theorems for the reformulated one. Those proofs will be given in Sects. 4-6.

Notations. We denote several positive constants depending on $a, b, \ldots$ by $C_{a, b}, \ldots$ or only by $C$ without confusion. We also denote $f(x) \sim g(x)$ as $x \rightarrow a$ when $C^{-1} g<f<C g$ in a neighborhood of $a$. For function spaces, $L^{2}$ denotes the space of square integrable functions on $\mathbf{R}$ with the norm

$$
\|f\|=\left(\int_{\mathbf{R}}|f(x)|^{2} d x\right)^{1 / 2} .
$$

Here and after the integrand $\mathbf{R}$ is abbreviated. $H^{l}(l \geqq 0)$ denotes the usual $l^{\text {th }}$ order Sobolev space with the norm

$$
\|f\|_{l}=\left(\sum_{j=0}^{l}\left\|\partial_{x}^{j} f\right\|^{2}\right)^{1 / 2}
$$


For the weight function $w, L_{w}^{2}$ denotes the space of measurable functions $f$ satisfying $\sqrt{w} f \in L^{2}$ with the norm

$$
|f|_{w}=\left(\int w(x)|f(x)|^{2} d x\right)^{1 / 2} .
$$

When $w(x)=\langle x\rangle^{\alpha}=\left(1+x^{2}\right)^{\alpha / 2}$, we write $L_{w}^{2}=L_{\alpha}^{2}$ and $|\cdot|_{w}=|\cdot|_{\alpha}$ without confusion. Moreover when $w$ is replaced by $\langle x\rangle^{\alpha} w$, we denote that space by $L_{\alpha, w}^{2}$ with the norm

$$
|f|_{\alpha, w}=\left(\int\langle x\rangle^{\alpha} w(x)|f(x)|^{2} d x\right)^{1 / 2} .
$$

When $C^{-1} \leqq w(x) \leqq C$, we note that $L^{2}=H^{0}=L_{0}^{2}=L_{w}^{2}$ with $\|\cdot\|=\|\cdot\|_{0}=$ $|\cdot|_{0} \sim|\cdot|_{w}$ and that $L_{\alpha, w}^{2}=L_{\alpha}^{2}$ with $|\cdot|_{\alpha, w} \sim|\cdot|_{\alpha}$.

\section{Preliminaries and Theorems}

We first state the existence of traveling wave solutions with shock profile. Substituting (1.4) into (1.1) we have

$$
\mu U_{\xi \xi}=-s U_{\xi}+f(U)_{\xi}=h^{\prime}(U) U_{\xi} .
$$

Integrating (2.1) over $( \pm \infty, \xi)$ and noting the Rankine-Hugoniot condition (1.5) we also have

$$
\mu U_{\xi}=-s\left(U-u_{ \pm}\right)+f(U)-f\left(u_{ \pm}\right)=h(U),
$$

which has a unique smooth solution $U$ up to a shift satisfying $U( \pm \infty)=u_{ \pm}$ provided that $h(U) \rightarrow 0$ at finite order as $U \rightarrow u_{ \pm}$. Moreover, if $f^{\prime}\left(u_{+}\right)<s<f^{\prime}\left(u_{-}\right)$, then $|h(U)| \sim\left|U-u_{ \pm}\right|$as $U \rightarrow u_{ \pm}$because of $h^{\prime}\left(u_{ \pm}\right) \neq 0$. Hence $\left|U(\xi)-u_{ \pm}\right| \sim$ $\exp (-c|\xi|)$ as $\xi \rightarrow \pm \infty$ for some constant $c$. When $s=f^{\prime}\left(u_{+}\right)$or $f^{\prime}\left(u_{-}\right),\left|U(\xi)-u_{ \pm}\right|$ $\sim|\xi|^{-1 / k_{ \pm}}$as $\xi \rightarrow \pm \infty$ provided $|h(U)| \sim\left|U-u_{ \pm}\right|^{1+k_{ \pm}}$for $k_{ \pm}>0$. We note $k_{ \pm}=n$ if $h^{\prime}\left(u_{ \pm}\right)=\cdots=h^{(n)}\left(u_{ \pm}\right)=0$ and $h^{(n+1)}\left(u_{ \pm}\right) \neq 0$. Thus we have

Lemma 1. Assume (1.5), (1.6) and

$$
|h(U)| \sim\left|U-u_{ \pm}\right|^{1+k_{ \pm}}, \quad U \rightarrow u_{ \pm}
$$

with $k_{ \pm} \geqq 0$. Then there exists a traveling wave solution $U(\xi)$ of (1.1) with $U( \pm \infty)=u_{ \pm}$, unique up to a shift, which is determined by the ordinary differential equation (2.1) or (2.2). Moreover, it holds as $\xi \rightarrow \pm \infty$,

$$
\begin{gathered}
\left|U(\xi)-u_{ \pm}\right| \sim \exp (-c|\xi|) \quad \text { if } \quad f^{\prime}\left(u_{+}\right)<s<f^{\prime}\left(u_{-}\right) \\
\left|U(\xi)-u_{+}\right| \sim|\xi|^{-/ k_{+}} \quad \text { if } \quad s=f^{\prime}\left(u_{+}\right),
\end{gathered}
$$

and

$$
\left|U(\xi)-u_{-}\right| \sim|\xi|^{-\mid k-} \text { if } s=f^{\prime}\left(u_{-}\right) .
$$

To investigate the stability of traveling wave solution $U$, we assume $u_{0}-U$ is integrable. First we determine a unique shift of $U$ as

$$
\int_{-\infty}^{\infty}\left(u_{0}(x)-U(x)\right) d x=0,
$$


and define

$$
\psi_{0}(x)=\int_{-\infty}^{x}\left(u_{0}(y)-U(y)\right) d y .
$$

Then our three theorems are given as follows.

Theorem 1 (Stability). Assume (1.5), (1.6) and (2.3) and let $U$ be a traveling wave solution uniquely determined by (2.7). Then the following hold:

(i) When $f^{\prime}\left(u_{+}\right)<s<f^{\prime}\left(u_{-}\right)$, suppose $u_{0}-U$ is integrable and $\psi_{0} \in H^{2}$. Then there exists a positive constant $\varepsilon_{1}$ such that if $\left\|\psi_{0}\right\|_{2}<\varepsilon_{1}$, then the Cauchy problem (1.1), (1.2) has a unique global solution $u(t, x)$ satisfying

$$
u-U \in C^{0}\left([0, \infty) ; H^{1}\right) \cap L^{2}\left(0, \infty ; H^{2}\right)
$$

and moreover

$$
\sup _{\mathbf{R}}|u(t, x)-U(x-s t)| \rightarrow 0 \text { as } t \rightarrow \infty .
$$

(ii) When $s=f^{\prime}\left(u_{+}\right)<f^{\prime}\left(u_{-}\right)$, there exists a positive constant $\varepsilon_{1}$ such that if $\left\|\psi_{0}\right\|_{2}$ $+\left|\psi_{0}\right|_{\langle\xi\rangle_{+}}<\varepsilon_{1}$ then the Cauchy problem (1.1),(1.2) has a unique global solution $u(t, x)$ satisfying

$$
u-U \in C^{0}\left([0, \infty) ; H^{1}\right) \cap L^{2}\left([0, \infty) ; H^{2} \cap L_{\langle\zeta\rangle_{+}}^{2}\right)
$$

and moreover

$$
\sup _{\mathbf{R}}|u(t, x)-U(x-s t)| \rightarrow 0 \text { as } t \rightarrow \infty,
$$

where $\langle\xi\rangle_{+}=\left\{\begin{array}{cc}\sqrt{1+\xi^{2}} & \xi \geqq 0 \\ 1 & \xi<0 .\end{array}\right.$

(iii) When $f^{\prime}\left(u_{+}\right)<s=f^{\prime}\left(u_{-}\right)$or $s=f^{\prime}\left(u_{+}\right)=f^{\prime}\left(u_{-}\right)$, then $L_{\langle\xi\rangle_{+}}^{2}$ in (ii) should be replaced by $L_{\langle\xi\rangle_{-}}^{2}$ or $L_{\langle\xi\rangle}^{2}=L_{1}^{2}$, respectively. Here $\langle\xi\rangle_{-}=\left\{\begin{array}{cc}\sqrt{1+\xi^{2}} & \xi<0 \\ 1 & \xi \geqq 0 .\end{array}\right.$

Remark 1. When $s=f^{\prime}\left(u_{+}\right)$or $f^{\prime}\left(u_{-}\right)$, we need a weight of order $\langle\xi\rangle=\sqrt{1+\xi^{2}}$ as $\xi \rightarrow+\infty$ or $-\infty$ for a stability theorem.

Theorem 2 (Rate of asymptotic speed for $f^{\prime}\left(u_{+}\right)<s<f^{\prime}\left(u_{-}\right)$). Let $u$ be a solution obtained in Theorem 1 (i) and let $\psi_{0}$ lie in $L_{\alpha}^{2}$ for some $\alpha>0$. If $\alpha$ is an integer, then it holds

$$
\sup _{\mathbf{R}}|u(t, x)-U(x-s t)| \leqq C(1+t)^{-\alpha / 2}\left(\left\|u_{0}-U\right\|_{1}+\left|\psi_{0}\right|_{\alpha}\right),
$$

while if $\alpha$ is not an integer, then

$$
\sup _{\mathbf{R}}|u(t, x)-U(x-s t)| \leqq C_{\varepsilon}(1+t)^{-\alpha / 2+\varepsilon}\left(\left\|u_{0}-U\right\|_{1}+\left|\psi_{0}\right|_{\alpha}\right)
$$

for any constant $\varepsilon>0$ and some constant $C_{\varepsilon}$ such that $C_{\varepsilon} \rightarrow \infty$ as $\varepsilon \rightarrow 0$.

Remark 2. For $f \in C^{2}$ Jones et al. [2] have shown $\sup _{\mathbf{R}}|u-U| \leqq C t^{-[\alpha] / 4}$ and, for $f^{\prime \prime}>0$, Kawashima and Matsumura [3] have shown $\sup _{\mathbf{R}}|u-U| \leqq C t^{-[\alpha] / 2}$. So, Theorems 1 and 2 improve both results. Further, for $f=u^{2} / 2$ Nishihara [7] showed, using an explicit formula, that if $\left|\psi_{0}(x)\right| \leqq C|x|^{-\alpha / 2}$ as $|x| \rightarrow \infty$, then 
$\sup _{\mathbf{R}}|u-U| \leqq C t^{-\alpha / 2}$ and this decay rate is optimal in general. Hence the decay rate in Theorem 2 seems to be almost optimal in the $L^{2}$-space version.

Next we state the result for $f^{\prime}\left(u_{+}\right)=s<f^{\prime}\left(u_{-}\right)$. When $f^{\prime}\left(u_{+}\right)<s=f^{\prime}\left(u_{-}\right)$or $s=f^{\prime}\left(u_{+}\right)=f^{\prime}\left(u_{-}\right)$, the similar result is obtained as in Theorem 1(iii).

Theorem 3 (Rate of asymptotic speed for $f^{\prime}\left(u_{+}\right)=s<f^{\prime}\left(u_{-}\right)$). Let $u$ be a solution obtained in Theorem 1(ii) and $f^{\prime \prime}\left(u_{+}\right)=\cdots=f^{(n)}\left(u_{+}\right)=0$ and $f^{(n+1)}\left(u_{+}\right) \neq 0$ for $n \geqq 1$. Then, if $\psi_{0} \in L_{\alpha,\langle\xi\rangle+}^{2}(0<\alpha<2 / n)$, it holds for any $\varepsilon>0$,

$$
\sup _{\mathbf{R}}|u(t, x)-U(x-s t)| \leqq C_{\varepsilon}(1+t)^{-\alpha / 4+\varepsilon}\left(\left\|u_{0}-U\right\|_{1}+\left|\psi_{0}\right|_{\alpha,\langle\xi\rangle+}\right) .
$$

Remark 3. Our rate seems to be not so sharp. But, the rate in this case is not known as far as we know. More contributions will be expected.

\section{Reformulation of the Problem}

Letting $U(\xi)$ be the traveling wave solution in Theorem 1, we put

$$
u(t, x)=U(\xi)+\psi_{\xi}(t, \xi), \quad \xi=x-s t
$$

Then the problem (1.1), (1.2) is reduced to

$$
\begin{aligned}
& \psi_{t}-s \psi_{\xi}+f\left(U+\psi_{\xi}\right)-f(U)=\mu \psi_{\xi \xi} \\
& \psi(0, \xi)=\psi_{0}(\xi) \equiv \int_{-\infty}^{\xi}\left(u_{0}-U\right)(\eta) d \eta
\end{aligned}
$$

Equation (3.2) is rewritten as

$$
\begin{gathered}
\psi_{t}+h^{\prime}(U) \psi_{\xi}-\mu \psi_{\xi \xi}=F, \\
F \equiv-\left(f\left(U+\psi_{\xi}\right)-f(U)-f^{\prime}(U) \psi_{\xi}\right) .
\end{gathered}
$$

Now we select the weight as

$$
w=w(U)=\left|\frac{\left(U-u_{+}\right)\left(U-u_{-}\right)}{h(U)}\right| .
$$

Since $w(U) \sim$ const. for the case $f^{\prime}\left(u_{+}\right)<s<f^{\prime}\left(u_{-}\right), L_{\alpha, w(U)}^{2}=L_{\alpha}^{2}$. While if $s=$ $f^{\prime}\left(u_{+}\right)<f^{\prime}\left(u_{-}\right)$, then $w(U) \sim|U-u|^{-k_{+}}$as $U \rightarrow u_{+}$and $w(U(\xi)) \sim\langle\xi\rangle$ as $\xi \rightarrow+\infty$, and hence $L_{w(U)}^{2}=L_{\langle\xi\rangle_{+}}^{2}$. Also, $L_{w(U)}^{2}=L_{\langle\xi\rangle_{-}}^{2}$ if $f^{\prime}\left(u_{+}\right)<f^{\prime}\left(u_{-}\right)=s$ and $L_{w(U)}^{2}=$ $L_{\langle\xi\rangle}^{2}=L_{1}^{2}$ if $f^{\prime}\left(u_{+}\right)=f^{\prime}\left(u_{-}\right)=s$. Noting these we define the solution space of (3.2) and (3.3),

$$
X(0, T)=\left\{\psi \in C^{0}\left([0, T] ; H^{2} \cap L_{w(U)}^{2}\right), \psi_{\xi} \in L^{2}\left(0, T ; H^{2} \cap L_{w(U)}^{2}\right)\right\},
$$

with $0<T \leqq+\infty$. Then the problem (3.2), (3.3) can be solved globally in time as follows.

Theorem 3.1. Suppose $\psi_{0} \in H^{2} \cap L_{w(U)}^{2}$. Then there exists a positive constant $\varepsilon_{2}$ such that if $\left\|\psi_{0}\right\|_{2}+\left|\psi_{0}\right|_{w(U)}<\varepsilon_{2}$, the problem (3.2), (3.3) has a unique global solution $\psi \in X(0, \infty)$ satisfying

$$
\|\psi(t)\|_{2}^{2}+|\psi(t)|_{w(U)}^{2}+\int_{0}^{t}\left(\left\|\psi_{\xi}(\tau)\right\|_{2}^{2}+\left|\psi_{\xi}(\tau)\right|_{w(U)}^{2}\right) d \tau \leqq C\left(\left\|\psi_{0}\right\|_{2}^{2}+\left|\psi_{0}\right|_{w(U)}^{2}\right)
$$


for any $t \geqq 0$. Moreover, $\psi_{\xi}$ tends to 0 in the maximum norm as $t \rightarrow \infty$, that is,

$$
\sup _{\mathbf{R}}\left|\psi_{\xi}(t, \xi)\right| \rightarrow 0 \text { as } t \rightarrow \infty \text {. }
$$

For the decay rate we have the following.

Theorem $3.2\left(f^{\prime}\left(u_{+}\right)<s<f^{\prime}\left(u_{-}\right)\right)$. Suppose $f^{\prime}\left(u_{+}\right)<s<f^{\prime}\left(u_{-}\right)$. Then the solution $\psi(t)$ obtained in Theorem 3.1 satisfies

$$
(1+t)^{\gamma}\|\psi(t)\|_{2}^{2}+\int_{0}^{t}(1+\tau)^{\gamma}\left\|\psi_{\xi}(\tau)\right\|_{2}^{2} d \tau \leqq C\left(\left|\psi_{0}\right|_{\alpha}^{2}+\left\|\psi_{0}\right\|_{2}^{2}\right)
$$

for any $\gamma$ such that $0 \leqq \gamma \leqq \alpha$ if $\alpha$ is an integer and that $0 \leqq \gamma<\alpha$ if $\alpha$ is not an integer.

Theorem $3.3\left(s=f^{\prime}\left(u_{+}\right)<f^{\prime}\left(u_{-}\right)\right)$. Suppose $s=f^{\prime}\left(u_{+}\right)<f^{\prime}\left(u_{-}\right)$and $f^{\prime \prime}\left(u_{+}\right)=\cdots=$ $f^{(n)}\left(u_{+}\right)=0$ and $f^{(n+1)}\left(u_{+}\right) \neq 0$ for $n \geqq 1$. If $0<\alpha<2 / n$, then the solution $\psi(t, x)$ obtained in Theorem 3.1 satisfies

$$
(1+t)^{\gamma}\|\psi(t)\|_{2}^{2}+\int_{0}^{t}(1+\tau)^{\gamma}\left\|\psi_{\xi}(\tau)\right\|_{2}^{2} d \tau \leqq C\left(\left\|\psi_{0}\right\|_{2}^{2}+\left|\psi_{0}\right|_{\alpha, w(U)}^{2}\right)
$$

for $\gamma$ such that $0 \leqq \gamma<\alpha / 2$.

All assertions (i)-(iii) in Theorem 1 are direct consequences of Theorem 3.1. Theorem 2 and Theorem 3 are, respectively, consequences of Theorem 3.2 and Theorem 3.3. Theorems 3.1-3.3 are all proved by a weighted energy method combining the local existence with a priori estimates. These are on the same line in Kawashima and Matsumura [3] etc.

Proposition 3.1 (Local existence). For any $\varepsilon_{0}>0$, there exists a positive constant $T_{0}$ depending on $\varepsilon_{0}$ such that if $\psi_{0} \in H^{2} \cap L_{w(U)}^{2}$ and $\left\|\psi_{0}\right\|_{2} \leqq \varepsilon_{0}$, then the problem (3.2), (3.3) has a unique solution $\psi \in X\left(0, T_{0}\right)$ satisfying $\|\psi(t)\|_{2}<2 \varepsilon_{0}$ for $0 \leqq t \leqq T_{0}$.

Proposition 3.2 (A priori estimate). Let $\psi$ be a solution in $X(0, T)$ for a positive constant $T$. Then there exists a positive constant $\varepsilon_{3}$ such that if $\sup _{0 \leqq t \leqq T}\|\psi(t)\|_{2}<\varepsilon_{3}$, then $\psi(t)$ satisfies (3.7) for $0 \leqq t \leqq T$.

Proposition 3.1 can be proved in the standard way. So we omit its proof. Proposition 3.2 will be proved in the next section. For the proofs of Theorems 3.2 and 3.3 more estimates are necessary.

In later sections we only show the case $u_{+}<u_{-}$and $h(U) \leqq 0$ for $U \in\left[u_{+}, u_{-}\right]$. The other case is shown in the same way.

\section{Basic Estimate and Stability Theorem}

Assuming $u_{+}<u_{-}$and $h(U)<0$ for $U \in\left(u_{+}, u_{-}\right)$, we first derive the basic estimate in all our proofs.

Lemma 4.1. Let $\psi(t) \in X(0, T)$ be a solution of (3.2), (3.3). Then it holds

$$
\frac{1}{2}|\psi(t)|_{w(U)}^{2}+\int_{0}^{t}\left(\left\|\sqrt{-U_{\xi}} \psi(\tau)\right\|^{2}+\mu\left|\psi_{\xi}(\tau)\right|_{w(U)}^{2}\right) d \tau \leqq \frac{1}{2}\left|\psi_{0}\right|_{w(U)}^{2}+\int_{0}^{t} \int_{0} w(U) \psi F d x d \tau .
$$


Proof. Multiplying (3.4) by $w(U(\xi)) \psi(t, \xi)$ we have

$$
\begin{aligned}
\left(\frac{1}{2} w(U) \psi^{2}\right)_{t} & +\left(\frac{1}{2}(w h)^{\prime}(U) \psi^{2}-\mu w(U) \psi_{\xi} \psi\right)_{\xi}+\mu w(U) \psi_{\xi}^{2} \\
& -\frac{1}{2}(w h)^{\prime \prime}(U) U_{\xi} \psi^{2}=w(U) \psi F .
\end{aligned}
$$

Here we have used $\mu U_{\xi}=h(U)$. Since we have taken the weight $w$ as (3.6), we obtain (4.1) by integrating (4.2) over $(0, t) \times \mathbf{R}$ and noting $U_{\xi}<0$. Q.E.D.

We now put

$$
N(t)=\sup _{0 \leqq \tau \leqq t}\|\psi(\tau)\|_{2},
$$

and assume $N(t) \leqq \varepsilon_{0}$. Since $|\psi| \leqq N(t),|F| \leqq C \psi_{\xi}^{2}$. Hence, if $N(t)<\varepsilon_{3}$ for sufficiently small $\varepsilon_{3}>0$, then we have

$$
|\psi(t)|_{w(U)}^{2}+\int_{0}^{t}\left|\psi_{\xi}(\tau)\right|_{w(U)}^{2} d \tau \leqq C\left|\psi_{0}\right|_{w(U)}^{2} .
$$

Moreover, we apply $\partial_{\xi}$ to (3.4), multiply it by $\partial_{\xi} \psi$ and $\partial_{\xi}^{3} \psi$ and integrate the resulting equations over $(0, t) \times \mathbf{R}$. Noting $\left|F_{\xi}\right| \leqq o(1)\left|\psi_{\xi}\right|+C\left|\psi_{\xi} \psi_{\xi \xi}\right|$ as $\sup _{\mathbf{R}}\left|\psi_{\xi}\right| \rightarrow 0$ we can get the next lemma. We omit the details.

Lemma 4.2. There is a positive constant $\varepsilon_{4}\left(\leqq \varepsilon_{0}\right)$ such that if $N(t) \leqq \varepsilon_{4}$, the estimate holds:

$$
\left\|\psi_{\xi}(t)\right\|_{1}^{2}+\int_{0}^{t}\left\|\psi_{\xi \xi}(\tau)\right\|_{1}^{2} d \tau \leqq C\left(\left|\psi_{0}\right|_{w(U)}^{2}+\left\|\psi_{0 \xi}\right\|_{1}^{2}\right)
$$

Combining Lemma 4.2 with (4.3) gives the proof of Proposition 3.2 and so Theorem 3.1.

\section{Decay Rate for the Case $f^{\prime}\left(u_{+}\right)<s<f^{\prime}\left(u_{-}\right)$}

We proceed to more a priori estimates of the solution $\psi$ of the problem (3.2), (3.3). Since $h(U)<0, U \in\left(u_{+}, u_{-}\right)$, there exists a unique number $\xi_{*} \in \mathbf{R}$ such that

$$
U\left(\xi_{*}\right)=\bar{u} \equiv \frac{u_{+}+u_{-}}{2}
$$

Putting $\left\langle\xi-\xi_{*}\right\rangle=\sqrt{1+\left(\xi-\xi_{*}\right)^{2}}$ and multiplying (3.2) by $2(1+t)^{\gamma}\left\langle\xi-\xi_{*}\right\rangle^{\beta} w(U) \psi$, we get

$$
\begin{aligned}
& \left((1+t)^{\gamma}\left\langle\xi-\xi_{*}\right\rangle^{\beta} w(U) \psi^{2}\right)_{t}+(\cdots)_{\xi}+2 \mu(1+t)^{\gamma}\left\langle\xi-\xi_{*}\right\rangle^{\beta} w(U) \psi_{\xi}^{2} \\
& \quad-\gamma(1+t)^{\gamma-1}\left\langle\xi-\xi_{*}\right\rangle^{\beta} w(U) \psi^{2}+(1+t)^{\gamma}\left\langle\xi-\xi_{*}\right\rangle^{\beta-1} A_{\beta} \psi^{2} \\
& \quad+2 \mu \beta(1+t)^{\gamma}\left\langle\xi-\xi_{*}\right\rangle^{\beta-2}\left(\xi-\xi_{*}\right) w(U) \psi \psi_{\xi} \\
& =2(1+t)^{\gamma}\left\langle\xi-\xi_{*}\right\rangle^{\beta} w(U) \psi F,
\end{aligned}
$$


where

by virtue of (3.6).

$$
\begin{aligned}
A_{\beta}(\xi) & =-\left\langle\xi-\xi_{*}\right\rangle U_{\xi}(w h)^{\prime \prime}(U)-\beta \frac{\xi-\xi_{*}}{\left\langle\xi-\xi_{*}\right\rangle}(w h)^{\prime}(U) \\
& =-2\left\langle\xi-\xi_{*}\right\rangle U_{\xi}-2 \beta \frac{\xi-\xi_{*}}{\left\langle\xi-\xi_{*}\right\rangle}(U-\bar{u})
\end{aligned}
$$

Lemma 5.1. Let $\alpha$ be a given positive number. For $\beta \in[0, \alpha]$, there is a positive number $c_{0}$ independent of $\beta$ such that

$$
A_{\beta}(\xi) \geqq c_{0} \beta \quad \text { for any } \xi \in \mathbf{R} .
$$

Proof. Let $g(\xi)=-(w h)^{\prime}(U(\xi))=-2(U(\xi)-\bar{u})$, then $g\left(\xi_{*}\right)=0$ by $(5.1)$ and $g^{\prime}(\xi)=$ $-2 U^{\prime}(\xi)>0$, so $g(\xi) \rightarrow u_{\mp}-u_{ \pm}$as $\xi \rightarrow \pm \infty$, respectively. Hence

$$
-\frac{\xi-\xi_{*}}{\left\langle\xi-\xi_{*}\right\rangle}(w h)^{\prime}(U(\xi)) \geqq c(\delta), \quad\left|\xi-\xi_{*}\right| \geqq \delta
$$

for any $\delta>0$. On the other hand,

$$
\begin{aligned}
-\left\langle\xi-\xi_{*}\right\rangle U_{\xi}(w h)^{\prime \prime}(U) & =-2\left\langle\xi-\xi_{*}\right\rangle U_{\xi}=2\left\langle\xi-\xi_{*}\right\rangle \frac{-h(U(\xi))}{\mu} \\
& \geqq \frac{-h(\bar{u})}{\mu}, \quad\left|\xi-\xi_{*}\right| \leqq \delta_{0}
\end{aligned}
$$

for some constant $\delta_{0}$. Combining (5.4) with (5.5) we obtain (5.3), where $c_{0}=$ $\min \left\{c\left(\delta_{0}\right), \frac{-h(\bar{u})}{\mu \alpha}\right\}$. Q.E.D.

Integrating (5.2) over $[0, t] \times \mathbf{R}$ and noting $C^{-1} \leqq w(U) \leqq C$, we have

$$
\begin{aligned}
& (1+t)^{\gamma}|\psi(t)|_{\beta}^{2}+\beta \int_{0}^{t}(1+\tau)^{\gamma}|\psi(\tau)|_{\beta-1}^{2} d \tau+\int_{0}^{t}(1+\tau)^{\gamma}\left|\psi_{\xi}(\tau)\right|_{\beta}^{2} d \tau \\
& \leqq \\
& \quad\left\{\left|\psi_{0}\right|_{\beta}^{2}+\gamma \int_{0}^{t}(1+\tau)^{\gamma-1}|\psi(\tau)|_{\beta}^{2} d \tau\right. \\
& +\beta \int_{0}^{t}(1+\tau)^{\gamma} \int\left\langle\xi-\xi_{*}\right\rangle^{\beta-1}\left|\psi \psi_{\xi}\right| d \xi d \tau \\
& \left.+\int_{0}^{t} \int(1+\tau)^{\gamma}\left\langle\xi-\xi_{*}\right\rangle^{\beta}|\psi||F| d \xi d \tau\right\}
\end{aligned}
$$

Since

$$
C \beta\left\langle\xi-\xi_{*}\right\rangle^{\beta-1}\left|\psi \psi_{\xi}\right| \leqq \frac{\beta}{2}\left\langle\xi-\xi_{*}\right\rangle^{\beta-1} \psi^{2}+\frac{C^{2} \beta}{2}\left\langle\xi-\xi_{*}\right\rangle^{\beta-1} \psi_{\xi}^{2}
$$


and

$$
\begin{aligned}
& \int \frac{C^{2} \beta}{2}\left\langle\xi-\xi_{*}\right\rangle^{\beta-1} \psi_{\xi}^{2} d \xi \\
& \quad=\int_{\left|\xi-\xi_{*}\right|>R} \frac{C^{2} \beta}{2\left\langle\xi-\xi_{*}\right\rangle}\left\langle\xi-\xi_{*}\right\rangle^{\beta} \psi_{\xi}^{2} d \xi+\int_{\left|\xi-\xi_{*}\right| \leqq R} \frac{C^{2} \beta}{2}\left\langle\xi-\xi_{*}\right\rangle^{\beta-1} \psi_{\xi}^{2} d \xi \\
& \quad \leqq \frac{1}{2}\left|\psi_{\xi}\right|_{\beta}^{2}+\beta C_{R}\left\|\psi_{\xi}\right\|^{2} .
\end{aligned}
$$

For some fixed $R>0$, we have

$$
\begin{aligned}
& (1+t)^{\gamma}|\psi(t)|_{\beta}^{2}+\int_{0}^{t}\left\{\frac{\beta}{2}(1+\tau)^{\gamma}|\psi(\tau)|_{\beta-1}^{2}+\left(\frac{1}{2}-C N(\tau)\right)(1+\tau)^{\gamma}\left|\psi_{\xi}(\tau)\right|_{\beta}^{2}\right\} d \tau \\
& \leqq C\left\{\left|\psi_{0}\right|_{\beta}^{2}+\gamma \int_{0}^{t}(1+\tau)^{\gamma-1}|\psi(\tau)|_{\beta}^{2} d \tau+\beta \int_{0}^{t}(1+\tau)^{\gamma}\left\|\psi_{\xi}\right\|^{2} d \tau\right\} .
\end{aligned}
$$

Thus we get the following.

Lemma 5.2. There is a positive constant $\varepsilon_{5}$ such that if $N(T)<\varepsilon_{5}$, then it holds for $t \in[0, T]$,

$$
\begin{aligned}
& (1+t)^{\gamma}|\psi(t)|_{\beta}^{2}+\int_{0}^{t}\left\{\beta(1+\tau)^{\gamma}|\psi(\tau)|_{\beta-1}^{2}+(1+\tau)^{\gamma}\left|\psi_{\xi}(\tau)\right|_{\beta}^{2}\right\} d \tau \\
& \quad \leqq C\left\{\left|\psi_{0}\right|_{\beta}^{2}+\gamma \int_{0}^{t}(1+\tau)^{\gamma-1}|\psi(\tau)|_{\beta}^{2} d \tau+\beta \int_{0}^{t}(1+\tau)^{\gamma}\left\|\psi_{\xi}(\tau)\right\|^{2} d \tau\right\} .
\end{aligned}
$$

Applying the induction to (5.7) we have

Lemma 5.3. It holds for $k=0,1, \ldots,[\alpha]$,

$$
\begin{gathered}
(1+t)^{k}|\psi(t)|_{\alpha-k}^{2}+\int_{0}^{t}\left\{(\alpha-k)(1+\tau)^{k}|\psi(\tau)|_{\alpha-k-1}^{2}\right. \\
\left.+(1+\tau)^{k}\left|\psi_{\xi}(\tau)\right|_{\alpha-k}^{2}\right\} d \tau \leqq C\left|\psi_{0}\right|_{\alpha}^{2} .
\end{gathered}
$$

Consequently, if $\alpha$ is an integer, then the following estimate holds for $0 \leqq \gamma \leqq \alpha$ :

$$
(1+t)^{\gamma}\|\psi(t)\|^{2}+\int_{0}^{t}(1+\tau)^{\gamma}\left\|\psi_{\xi}(\tau)\right\|^{2} d \tau \leqq C\left|\psi_{0}\right|_{\alpha}^{2} .
$$

Proof. First, letting $\gamma=0$ and $\beta=\alpha$ in (5.7) we have (5.8) , which shows the lemma for $\alpha<1$. Here we have used (4.3). Next we take $1 \leqq \alpha<2$. Letting $\beta=0$ and $\gamma=1$ and letting $\beta=\alpha-1$ and $\gamma=1$ in (5.7) show (5.8) $)_{1}$. Hence the proof for $\alpha<2$ is completed. Repeating the same procedure we can get the desired estimate $(5.8)_{k}$ for any $\alpha \geqq 0$. Q.E.D.

Methods used in this section till now are almost same as in Kawashima and Matsumura [3]. Further we show a sharper estimate. Let $\alpha$ be not an integer and $\gamma$ be $[\alpha]<\gamma<\alpha$. Taking $\beta=0$ in (5.7) we have

$$
(1+t)^{\gamma}|\psi(t)|_{0}^{2}+\int_{0}^{t}(1+\tau)^{\gamma}\left|\psi_{\xi}(\tau)\right|_{0}^{2} d \tau \leqq C\left(\left|\psi_{0}\right|_{0}^{2}+\gamma \int_{0}^{t}(1+\tau)^{\gamma-1}|\psi(\tau)|_{0}^{2} d \tau\right) .
$$


Using $(5.8)_{k}$ with $k=[\alpha]$,

$$
\begin{aligned}
& (1+t)^{[\alpha]}|\psi(t)|_{\alpha-[\alpha]}^{2}+\int_{0}^{t}\left\{(\alpha-[\alpha])(1+\tau)^{[\alpha]}|\psi(\tau)|_{\alpha-[\alpha]-1}^{2}\right. \\
& \left.+(1+\tau)^{[\alpha]}\left|\psi_{\xi}(\tau)\right|_{\alpha}^{2}\right\} d \tau \leqq C\left|\psi_{0}\right|_{\alpha}^{2},
\end{aligned}
$$

we estimate the final term in (5.10):

$$
\begin{aligned}
\int_{0}^{t}(1+\tau)^{\gamma-1}|\psi(\tau)|_{0}^{2} d \tau \\
\quad=\int_{0}^{t}(1+\tau)^{\gamma-1} \int\left\langle\xi-\xi_{*}\right\rangle^{(\alpha-[\alpha])([\alpha]+1-\alpha)-(\alpha-[\alpha])([\alpha]+1-\alpha)}\left(\psi^{2}\right)^{([\alpha]+1-\alpha)+(\alpha-[\alpha])} d \xi d \tau \\
\quad \leqq \int_{0}^{t}(1+\tau)^{\gamma-1}\left(\int\left\langle\xi-\xi_{*}\right\rangle^{\alpha-[\alpha]} \psi^{2} d \xi\right)^{[\alpha]+1-\alpha}\left(\int\left\langle\xi-\xi_{*}\right\rangle^{-([\alpha]+1-\alpha)} \psi^{2} d \xi\right)^{\alpha-[\alpha]} d \tau \\
\quad=\int_{0}^{t}(1+\tau)^{-([\alpha]+1-\gamma)}\left((1+\tau)^{[\alpha]}|\psi|_{\alpha-[\alpha]}^{2}\right)^{[\alpha]+1-\alpha}\left((1+\tau)^{[\alpha]}|\psi|_{\alpha-[\alpha]-1}^{2}\right)^{\alpha-[\alpha]} d \tau \\
\quad \leqq C\left|\psi_{0}\right|_{\alpha}^{2([\alpha]+1-\alpha)} \int_{0}^{t}(1+\tau)^{-([\alpha]+1-\gamma)}\left((1+\tau)^{[\alpha]}|\psi|_{\alpha-[\alpha]-1}^{2}\right)^{\alpha-[\alpha]} d \tau \\
\quad \leqq C\left|\psi_{0}\right|_{\alpha}^{2([\alpha]+1-\alpha)}\left(\int_{0}^{t}(1+\tau)^{-\frac{[\alpha]+1-\gamma}{[\alpha]+1-\alpha}} d \tau\right)^{[\alpha]+1-\alpha}\left(\int_{0}^{t}(1+\tau)^{[\alpha]}|\psi|_{\alpha-[\alpha]-1}^{2} d \tau\right)^{\alpha-[\alpha]} \\
\quad \leqq C\left|\psi_{0}\right|_{\alpha}^{2},
\end{aligned}
$$

because of $\frac{[\alpha]+1-\gamma}{[\alpha]+1-\alpha}>1$. Thus we have the following from (5.10).

Lemma 5.4. If $\alpha$ is not an integer, then it holds for any given $\gamma<\alpha$,

$$
(1+t)^{\gamma}\|\psi(t)\|^{2}+\int_{0}^{t}(1+\tau)^{\gamma}\left\|\psi_{\xi}(\tau)\right\|^{2} d \tau \leqq C\left|\psi_{0}\right|_{\alpha}^{2} .
$$

Similar estimates to Lemma 4.2 give the same decay rate for derivatives of the solution. The details are omitted.

Lemma 5.5. Let $l=1$ and 2. For any $\gamma \leqq \alpha(\alpha$ :integer $)$ or $\gamma<\alpha(\alpha:$ not integer $)$, there exists a positive constant $\varepsilon_{6}$ such that if $N(T) \leqq \varepsilon_{6}$, then the decay estimate

$$
(1+t)^{\gamma}\left\|\partial_{\xi}^{l} \psi(t)\right\|^{2}+\int_{0}^{t}(1+\tau)^{\gamma}\left\|\partial_{\xi}^{l+1} \psi(\tau)\right\|^{2} d \tau \leqq C\left(\left\|\psi_{0}\right\|_{2}^{2}+\left|\psi_{0}\right|_{\alpha}^{2}\right) .
$$

Combining the latter part of Lemma 5.3, Lemma 5.4 with Lemma 5.5 we complete the proof of Theorem 3.2.

\section{Decay Rate for the Case $s=f^{\prime}\left(u_{+}\right)<f^{\prime}\left(u_{-}\right)$}

First we show the following estimate for the solution $\psi$ obtained in Theorem 3.1. 
Lemma 6.1. For $0<\beta \leqq \alpha<2 / n(n \geqq 1)$, there exists a positive constant $\varepsilon_{7}$ such that if $N(T) \leqq \varepsilon_{7}$, then the estimate

$$
\begin{aligned}
& \int w(U)^{1+\beta} \psi(t, \xi)^{2} d \xi+\int_{0}^{t} \int_{\xi>0} w(U)^{\beta-1} \psi(\tau, \xi)^{2} d \xi d \tau \\
& \quad+\int_{0}^{t} \int w(U)^{1+\beta} \psi_{\xi}(\tau, \xi)^{2} d \xi d \tau \leqq C \int w(U)^{1+\beta} \psi_{0}(\xi)^{2} d \xi .
\end{aligned}
$$

Proof. Letting $z(\xi)$ be a positive function and multiplying (3.4) by $2 z w(U) \psi$, we have

$$
\begin{gathered}
\left(z w(U) \psi^{2}\right)_{t}+(\cdots)_{\xi}+2 \mu z w(U) \psi_{\xi}^{2}-2\left(z(h w)^{\prime}(U)\right)_{\xi} \psi^{2} \\
+2 \mu z_{\xi} w(U) \psi \psi_{\xi}=2 z w(U) \psi F
\end{gathered}
$$

Since

$$
-\left(z(h w)^{\prime}(U)\right)_{\xi}=2(\bar{u}-U) z_{\xi}-2 z U_{\xi}
$$

and

$$
\left|2 \mu z_{\xi} w(U) \psi \psi_{\xi}\right| \leqq 2 \varepsilon \mu z w(U) \psi_{\xi}^{2}+\frac{\mu w(U) z_{\xi}^{2}}{2 \varepsilon z} \psi^{2}
$$

for $\varepsilon \in(0,1)$, Eq. (6.2) yields

$$
\begin{aligned}
\left(z w(U) \psi^{2}\right)_{t} & +(\cdots)_{\xi}+2(1-\varepsilon) \mu z w(U) \psi_{\xi}^{2}+2\left\{-2 z U_{\xi}+\left(2(\bar{u}-U)-\frac{\mu w(U) z_{\xi}}{2 \varepsilon Z}\right) z_{\xi}\right\} \psi^{2} \\
& \leqq 2 z w(U)|\psi F| .
\end{aligned}
$$

Taking $z=w(U)^{\beta}$, we have

$$
I \equiv\left(2(\bar{u}-U)-\frac{\mu w(U) z_{\xi}}{2 \varepsilon Z}\right) z_{\xi}=\frac{\beta}{\mu} w(U)^{\beta-1} w^{\prime}(U) h(U)\left(2(\bar{u}-U)-\frac{\beta w^{\prime}(U) h(U)}{2 \varepsilon}\right) .
$$

If we put $\delta=U-u_{+}>0$ and $\tilde{u}=u_{-}-u_{+}$, then we have near $u_{+}$or $\xi=+\infty$,

$$
\begin{aligned}
h(U) w^{\prime}(U) & =2 \delta-\tilde{u}-\frac{\delta(\delta-\tilde{u}) h^{\prime}(U)}{h(U)} \\
& =2 \delta-\tilde{u}-\frac{\delta(\delta-\tilde{u})\left(h^{\prime \prime}\left(u_{+}\right) \delta+\cdots+\frac{h^{(n+1)}\left(u_{+}\right)}{n !} \delta^{n}+o\left(\delta^{n}\right)\right)}{\frac{h^{\prime \prime}\left(u_{+}\right)}{2 !} \delta^{2}+\cdots+\frac{h^{(n+1)}\left(u_{+}\right)}{(n+1) !} \delta^{n+1}+o\left(\delta^{n+1}\right)} \\
& =2 \delta-\tilde{u}-(\delta-\tilde{u})((n+1)+O(\delta)) \\
& =\tilde{u} n+O(\delta)
\end{aligned}
$$

and hence

$$
I=\frac{\beta w(U)^{\beta-1}}{\mu}(\tilde{u} n+O(\delta))\left(\tilde{u}\left(1-\frac{\beta n}{2 \varepsilon}\right)+O(\delta)\right)
$$


Since $\beta \leqq \alpha<\frac{2}{n}$, if we set $\varepsilon<1$ as $1-\frac{\beta n}{2 \varepsilon}>0$, then there are positive constants $c_{1}$ and $R_{1}$ such that

$$
I \geqq c_{1} \quad \text { for } \quad \xi \geqq R_{1} .
$$

Noting $C^{-1} \leqq w(U) \leqq C, C^{-1} \leqq w^{\prime}(U) \leqq C$ as $\xi \rightarrow-\infty$ and using Lemma 4.1, we have

$$
\int_{0}^{t} \int_{\xi \leqq R_{1}} 2 I \cdot \psi^{2} d \xi d \tau \leqq C\left|\psi_{0}\right|_{w(U)}^{2} .
$$

Because of (6.4) and (6.5) the integration of $(6.3)$ over $(0, t) \times \mathbf{R}$ gives the estimate (6.1). Q.E.D.

Again multiplying (3.2) by $2(1+t)^{\gamma}\left\langle\xi-\xi_{*}\right\rangle^{\beta} w(U) \psi$ and integrating its equation $(=(5.2))$ over $(0, t) \times \mathbf{R}$, we have for $0 \leqq \beta \leqq \alpha$,

$$
\begin{aligned}
& (1+t)^{\gamma}|\psi(t)|_{\beta, w(U)}^{2}+(1-C N(T)) \int_{0}^{t}(1+\tau)^{\gamma}\left|\psi_{\xi}(\tau)\right|_{\beta, w(U)}^{2} d \tau \\
& +\beta \int_{0}^{t}(1+\tau)^{\gamma}|\psi(\tau)|_{\beta-1}^{2} d \tau \\
& \leqq \\
& C\left\{\left|\psi_{0}\right|_{\beta, w(U)}^{2}+\gamma \int_{0}^{t}(1+\tau)^{\gamma-1}|\psi(\tau)|_{\beta, w(U)}^{2} d \tau\right. \\
& \left.+\beta \int_{0}^{t}(1+\tau)^{\gamma} \int\left\langle\xi-\xi_{*}\right\rangle^{\beta-1} w(U)\left|\psi \psi_{\xi}\right| d \xi d \tau\right\} .
\end{aligned}
$$

For $(6.6)_{\gamma, \beta}$ with $\gamma=0$ and $\beta \leqq \alpha$,

$$
\begin{aligned}
\mid \text { last term in }(6.6)_{0, \beta} \mid & \leqq \int_{0}^{t} \int_{\frac{\beta}{2}}\left\langle\xi-\xi_{*}\right\rangle^{\beta-1} \psi^{2} \\
& +\frac{C^{2} \beta}{2}\left\langle\xi-\xi_{*}\right\rangle^{\beta-1} w(U)^{2} \psi_{\xi}^{2} d \xi d \tau,
\end{aligned}
$$

and

$$
\begin{aligned}
& \frac{C^{2} \beta}{2} \int_{0}^{t} \int\left\langle\xi-\xi_{*}\right\rangle^{\beta-1} w(U)^{2} \psi_{\xi}^{2} d \xi d \tau \\
& \leqq C \int_{0}^{t} \int_{\xi>R_{2}} w(U)^{\beta+1} \psi_{\xi}^{2} d \xi d \tau+\frac{1}{2} \int_{0}^{t} \int_{\xi<-R_{3}}\left\langle\xi-\xi_{*}\right\rangle^{\beta} \psi_{\xi}^{2} d \xi d \tau \\
& \quad+C \int_{0}^{t} \int_{-R_{3} \leqq \xi \leqq R_{2}} \psi_{\xi}^{2} d \xi d \tau
\end{aligned}
$$

for some constants $R_{2}, R_{3}>0$, because $w(U(\xi)) \sim \xi$ as $\xi \rightarrow \infty$ and $w(U(\xi)) \sim$ const. as $\xi \rightarrow-\infty$. Applying Lemma 6.1 and Lemma 4.1 to $(6.6)_{0, \beta},(6.7)$ and $(6.8)$, and taking $\beta=\alpha$, we get the following. 
Lemma 6.2. There is a positive constant $\varepsilon_{7}$ such that if $N(T) \leqq \varepsilon_{7}$, then the estimate

$$
|\psi(t)|_{\alpha, w(U)}^{2}+\int_{0}^{t}\left(|\psi(\tau)|_{\alpha-1}^{2}+\left|\psi_{\xi}(\tau)\right|_{\alpha, w(U)}^{2}\right) d \tau \leqq C\left|\psi_{0}\right|_{\alpha, w(U)}^{2}
$$

holds for $\alpha<\frac{2}{n}(n \geqq 1)$.

Next we consider $(6.6)_{\gamma, \beta}$ with $\gamma<\alpha / 2$ and $\beta=0$ :

$$
\begin{aligned}
& (1+t)^{\gamma}|\psi(t)|_{0, w(U)}^{2}+(1-N(T)) \int_{0}^{t}(1+\tau)^{\gamma}\left|\psi_{\xi}(\tau)\right|_{0, w(U)}^{2} d \tau \\
& \leqq C\left(\left|\psi_{0}\right|_{0, w(U)}^{2}+\gamma \int_{0}^{t}(1+\tau)^{\gamma-1}|\psi(\tau)|_{0, w(U)}^{2} d \tau\right) .
\end{aligned}
$$

We estimate the final term in a similar fashion to the proof of Lemma 5.4. We divide the integrand as

$$
\begin{aligned}
& C \gamma \int_{0}^{t}(1+\tau)^{\gamma-1}|\psi(\tau)|_{0, w(U)}^{2} d \tau \\
& \quad \leqq C \int_{0}^{t}(1+\tau)^{\gamma-1}\left(\int_{\xi>0}+\int_{\xi<0}\right) w(U(\xi)) \psi(\tau, \xi)^{2} d \xi d \tau \\
& \quad \leqq C \int_{0}^{t}(1+\tau)^{\gamma-1} \int_{\xi>0} w(U) \psi(\tau, \xi)^{2} d \xi d \tau+C \int_{0}^{t}(1+\tau)^{\gamma-1} \int_{\xi<0} \psi(\tau, \xi)^{2} d \xi d \tau \\
& \quad \equiv I_{1}+I_{2} .
\end{aligned}
$$

Noting $\int_{\xi>0} w(U)^{1+\alpha} \psi^{2} d \xi \sim \int_{\xi>0}\left\langle\xi-\xi_{*}\right\rangle^{\alpha} w(U) \psi^{2} d \xi$, we have

$$
\begin{aligned}
I_{1} & \leqq C \int_{0}^{t}(1+\tau)^{\gamma-1}\left(\int_{\xi>0} w(U)^{1+\alpha} \psi^{2} d \xi\right)^{\frac{2-\alpha}{2}}\left(\int_{\xi>0} w(U)^{\alpha-1} \psi^{2} d \xi\right)^{\frac{\alpha}{2}} d \tau \\
& \leqq C\left|\psi_{0}\right|_{\alpha, w(U)}^{2-\alpha} \int_{0}^{t}(1+\tau)^{\gamma-1}\left(\int_{\xi>0} w(U)^{\alpha-1} \psi^{2} d \xi\right)^{\alpha / 2} d \tau \\
& \leqq C\left|\psi_{0}\right|_{\alpha, w(U)}^{2-\alpha}\left(\int_{0}^{t}(1+\tau)^{-\frac{2(1-\gamma)}{2-\alpha}} d \tau\right)^{\frac{2-\alpha}{2}}\left(\int_{0}^{t} \int_{\xi>0} w(U)^{\alpha-1} \psi^{2} d \xi d \tau\right)^{\frac{\alpha}{2}} \\
& \leqq C\left|\psi_{0}\right|_{\alpha, w(U)}^{2}
\end{aligned}
$$

by (6.1) $)_{\alpha}$ and (6.9) and $\frac{2(1-\gamma)}{2-\alpha}>1$. While, since $\gamma<\frac{\alpha}{2}<\frac{1}{n} \leqq 1$ by $n \geqq 1$,

$$
I_{2} \leqq C \int_{0}^{t} \int_{\xi<0} \psi(\tau, \xi)^{2} d \xi d \tau \leqq C\left|\psi_{0}\right|_{w(U)}^{2}
$$


by virtue of (6.9) provided $\alpha \geqq 1$ or $n=1$. Let $n \geqq 2$ and so $\alpha<1$. Then, by virtue of (6.9)

$$
\begin{aligned}
& I_{2} \leqq C \int_{0}^{t}(1+\tau)^{\gamma-1} \int_{\xi<0}\langle\xi\rangle^{\alpha(1-\alpha)+\alpha(\alpha-1)} \psi^{2} d \xi d \tau \\
& \leqq C \int_{0}^{t}(1+\tau)^{\gamma-1}\left(\int_{\xi<0}\langle\xi\rangle^{\alpha} \psi^{2} d \xi\right)^{1-\alpha}\left(\int_{\xi<0}\langle\xi\rangle^{\alpha-1} \psi^{2} d \xi\right)^{\alpha} d \tau \\
& \leqq C\left|\psi_{0}\right|_{\alpha, w(U)}^{2(1-\alpha)} \int_{0}^{t}(1+\tau)^{-(1-\gamma)}|\psi(\tau)|_{\alpha-1}^{2 \alpha} d \tau \\
& \leqq C\left|\psi_{0}\right|_{\alpha, w(U)}^{2(1-\alpha)}\left(\int_{0}^{t}(1+\tau)^{-\frac{1-\gamma}{1-\alpha} d \tau}\right)^{1-\alpha}\left(\int_{0}^{t}|\psi(\tau)|_{\alpha-1}^{2} d \tau\right)^{\alpha} \\
& \leqq C\left|\psi_{0}\right|_{\alpha, w(U)}^{2}
\end{aligned}
$$

because of $\frac{1-\gamma}{1-\alpha}>1$ by $\gamma<\alpha / 2$. Thus we have had a desired estimate.

Lemma 6.3. For $N(T) \leqq \varepsilon_{7}$, it holds for $\gamma<\alpha / 2<1 / n$,

$$
(1+t)^{\gamma}|\psi(t)|_{w(U)}^{2}+\int_{0}^{t}(1+\tau)^{\gamma}\left|\psi_{\xi}(\tau)\right|_{w(U)}^{2} d \tau \leqq C\left|\psi_{0}\right|_{\alpha, w(U)}^{2} .
$$

We complete the proof of Theorem 3.3 by obtaining the decay rate for derivatives of the solution, similar to Lemma 5.5. The details are omitted too.

Lemma 6.4. Let $l=1$ and $l=2$. There exists a positive constant $\varepsilon_{8}$ such that if $N(T) \leqq \varepsilon_{8}$, then the decay estimate

$$
(1+t)^{\gamma}\left\|\partial_{\xi}^{l} \psi(t)\right\|^{2}+\int_{0}^{t}(1+\tau)^{\gamma}\left\|\partial_{\xi}^{l+1} \psi(\tau)\right\|^{2} d \tau \leqq C\left(\left\|\psi_{0}\right\|_{2}^{2}+\left|\psi_{0}\right|_{\alpha, w(U)}^{2}\right)
$$

holds for any $\gamma, 0 \leqq \gamma<\alpha / 2<1 / n$.

\section{References}

1. Il'in, A.M., Oleinik, O.A.: Asymptotic behavior of the solutions of the Cauchy problem for certain quasilinear equations for large time (Russian). Mat. Sbornik 51, 191-216 (1960)

2. Jones, C., Gardner, R., Kapitula, T.: Stability of travelling waves for non-convex scalar viscous conservation laws. Commun. Pure Appl. Math. 46, 505-526 (1993)

3. Kawashima, S., Matsumura, A.: Asymptotic stability of traveling wave solutions of systems for one-dimensional gas motion. Commun. Math. Phys. 101, 97-127 (1985)

4. Kawashima, S., Matsumura, A.: Stability of shock profiles in viscoelasticity with non-convex constitutive relations. To appear

5. Lax, P.D.: Hyperbolic systems of conservation laws II. Commun. Pure Appl. Math. 10, 537-566 (1957)

6. Mei, M.: Stability of shock profiles for non-convex scalar viscous conservation laws. To appear

7. Nishihara, K.: A note on the stability of traveling wave solutions of Burgers' equation. Jpn. J. Appl. Math. 2, 27-35 (1985)

8. Osher, S., Ralston, J.: $L^{1}$ stability of travelling waves with applications to convective porous media flow. Commun. Pure Appl. Math. 35, 737-751 (1982)

9. Weinberger, H.F.: Long-time behavior for a regularized scalar conservation law in the absence of genuine nonlinearity. Ann. Inst. Henri Poincaré 7, 407-425 (1990)

Communicated by S.-T. Yau 